\title{
Mise au point aéroacoustique des véhicules chez PSA
}

\author{
Vehicles aeroacoustic perfecting at PSA
}

par L. Burgade

P.S.A. Peugeot Citroën

Due to the increasing importance of acoustic comfort in vehicles, car manufacturers have developed their knowledge and methodologies in automobile aeroacoustics. After having achieved a better understanding of theoretical aerodynamics and acoustics relationships, carmakers have applied it to settle new development methods and techniques. Thanks to these new measurement methods in new low background noise aeroacoustic windtunnels, great improvements have been obtained, particularly on outer devices like rearview mirrors, windshied wipers... Further developments will be done in two directions : computational aeroacoustics and psychoacoustics.

\section{IINTRODUCTION}

\section{1 importance du bruit aérodynamique}

Les bruits d'origine aérodynamique occupent une place de plus en plus importante dans le confort acoustique des véhicules. Cela s'explique par les raisons suivantes :

- l'exigence croissante de la clientèle en termes de confort acoustique,

- la réduction des bruits d'origine moteur,

- l'usage autoroutier de plus en plus fréquent en Europe et l'amélioration de la qualité du revêtement des routes.

Le bruit des véhicules provient de trois sources principales : le moteur, le contact sol-pneumatique (roulement) et l'écoulement de l'air autour de la voiture (aérodynamique).

On constate que les bruits d'origine aérodynamique sont subjectivement présents à partir de $100 \mathrm{~km} / \mathrm{h}$ et deviennent prédominants à partir de $140 \mathrm{~km} / \mathrm{h}$ si on considère le roulage sur un revêtement routier acceptable. Sur un revêtement du type enrobé drainant, les bruits de roulement sont bien plus faibles, et les bruits aérodynamiques prédominants dès $100 \mathrm{~km} / \mathrm{h}$. Le bruit aérodynamique représente ainsi une part majoritaire du niveau sonore en usage autoroutier. Il a beaucoup d'importance sur l'évolution de la vigilance et de la fatigue lors de longs trajets. Il est donc apparu qu'un effort particulier devait être porté sur ce thème et qu'il fallait faire progresser connaissances et méthodes de travail.
- 1.2 Zones du véhicule impliquées dans l'aéroacoustique

Les bruits intérieurs d'origine aérodynamique sont de trois types principaux (fig. 1):

- les bruits de forme liés au style et à l'intégration des accessoires extérieurs (rétroviseurs, essuie-vitres, antennes, etc.),

- les défauts d'étanchéité des ouvrants,

- les bruits de fentes et de circulation dans les corps creux (joint supérieur de pare-brise, feuillures de portières, etc.).

Ils pénètrent dans l'habitacle à travers tous les panneaux et en particulier les vitrages, mais aussi au niveau des contacts des joints sur la caisse, les ouvrants, et des coulisses et lécheurs sur les vitres latérales. Ils sont donc influencés par les paramètres suivants :

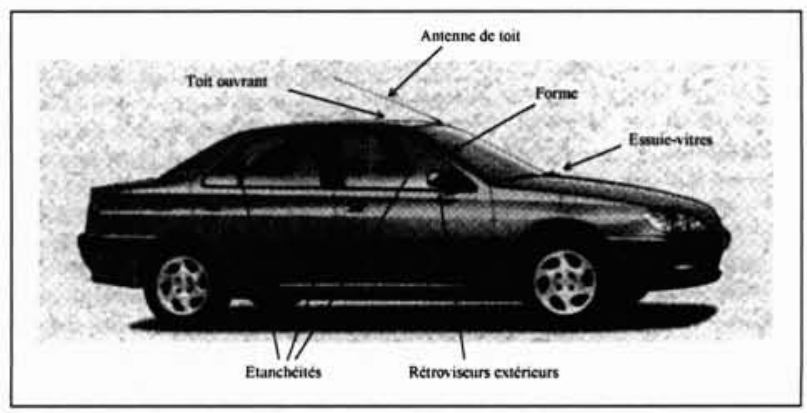

1. Zones du véhicule impliquées dans le bruit aérodynamique 
- l'isolation des vitrages,

- l'isolation des joints, des coulisses et lécheurs de vitres latérales.

- l'absorption des matériaux de garnissage dans l'habitacle.

L'aéroacoustique est donc un sujet de synthèse qui requiert une étude de tous ces sujets et un suivi de l'évolution de leur définition tout au long du développement des nouveaux véhicules (Cf. [1], [2] et [3]).

\section{MISE AU POINT AÉROACOUSTIQUE DU VÉHICULE}

\subsection{Analyse des différents types de bruits aérodyna- miques automobiles}

On peut regrouper les sujets à mettre au point en bruit aérodynamique automobile en quatre sous-ensembles :

- les étanchéités.

- les fentes et cavités.

- la forme du véhicule sans accessoire extérieur,

- les accessoires extérieurs.

Ces sujets relativement indépendants sont dans un premier temps étudiés séparément, puis analysés ensemble sur prototype.

\subsection{Processus de mise au point}

La mise au point expérimentale en aéroacoustique se fait en différentes étapes, sur différents supports et dans différents moyens d'essais. Dans le cadre de l'étude de synthèse aéroacoustique d'un nouveau véhicule, il est ainsi nécessaire d'étudier successivement les sujets suivants :

- estimation des efforts aérodynamiques sur les portières afin de bien en dimensionner la rigidité (aucune fuite ne devra apparaître sur le véhicule de série jusqu'à sa vitesse maximale pour des angles de dérapage compris entre - 10 et +10 degrés). On utilise alors des résultats d'essais en soufflerie ou de calculs aérodynamiques 3D,

- mise au point des joints de portières, des lécheurs et coulisses de vitres en étanchéité et en transparence quels que soient les réglages, les intervalles de tolérances et les efforts sur les portes,
- mise au point en soufflerie aéroacoustique du bruit de forme, des accessoires extérieurs, et des étanchéités sous efforts et excitation aérodynamiques,

- essais de synthèse sur prototypes.

\subsubsection{Mise au point des étanchéités}

Elle se fait sur deux types de moyens : la petite cabine et la chambre réverbérante (Cf. fig. 2). Le premier moyen d'essais sert à aider le choix du type de joint ; c'est un moyen partiel de petites dimensions. Le second permet de faire une synthèse des étanchéités sur tout un côté de caisse en présence et en l'absence d'efforts aérodynamiques. On peut en effet tirer en un certain nombre de points sur les portes afin de simuler le champs de pression sur ces dernières en condition de roulage.

Outre les étanchéités, le chambre réverbérante permet d'évaluer l'influence de modifications d'isolation de certains éléments tels que les doublures de portes, les vitrages... Elle permet ainsi de faire une analyse complète d'un côté de caisse avant même un essai en soufflerie aéroacoustique.

\subsubsection{Mise au point en soufflerie aéroacoustique}

\section{a) Principe}

L'évaluation du bruit aérodynamique se fait en plaçant le véhicule à étudier dans la veine d'essais d'une soufflerie aéroacoustique. On simule les conditions de roulage en soufflant sur la voiture. Le bruit intérieur généré est alors mesuré à l'aide de mannequins acoustiques possédant des micros au niveau des oreilles [3] positionnés dans l'habitacle aux places des passagers. Des mesures complémentaires peuvent également être réalisées dans l'écoulement afin de mieux caractériser les sources ([1], [3], [4] et [5]).

\section{b) Méthodologie}

Le cahier des charges acoustique global sert de point de départ à la détermination des cahiers des charges partiels pour chaque type de bruit, et en particulier pour ceux d'origine aérodynamique. L'objectif des essais est alors de comparer les résultats aux différents objectifs à atteindre afin de valider ou faire évoluer les définitions.

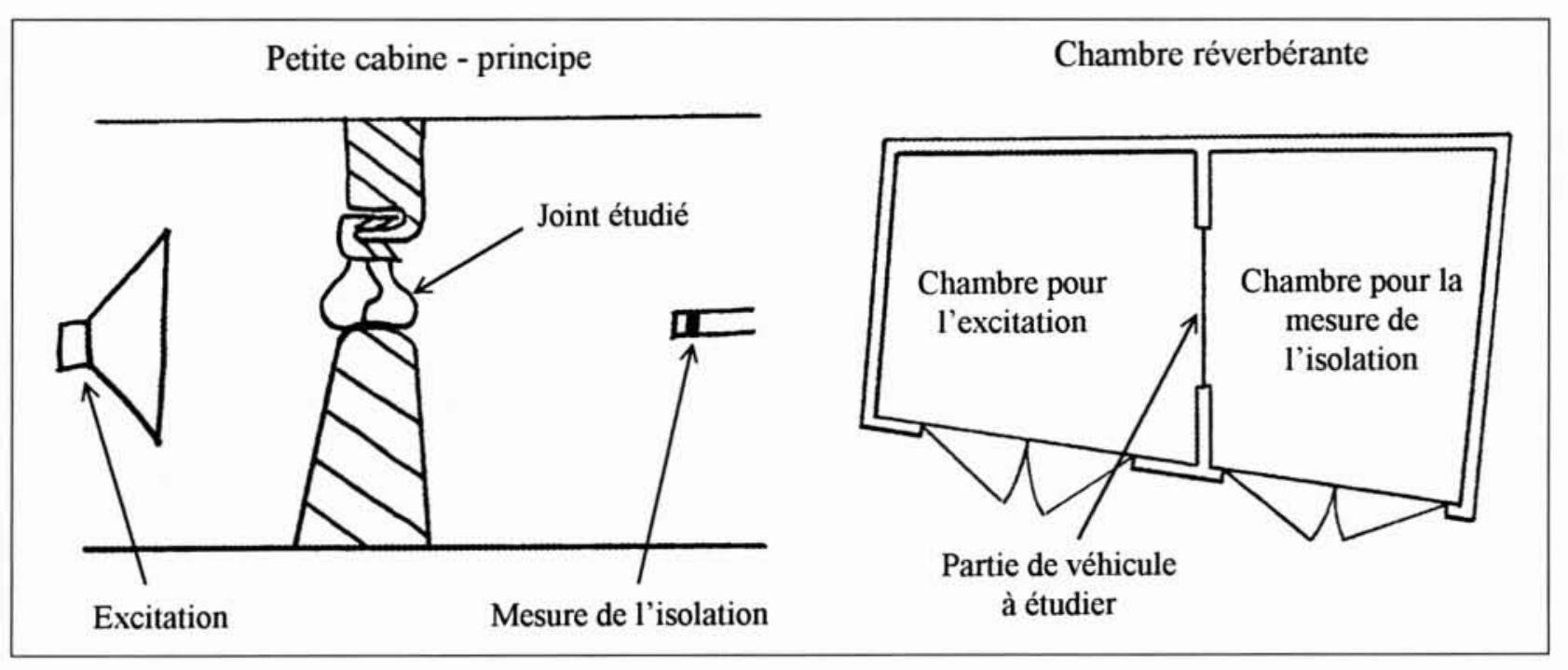

2. Schéma d'une petite cabine et d'une chambre réverbérante 


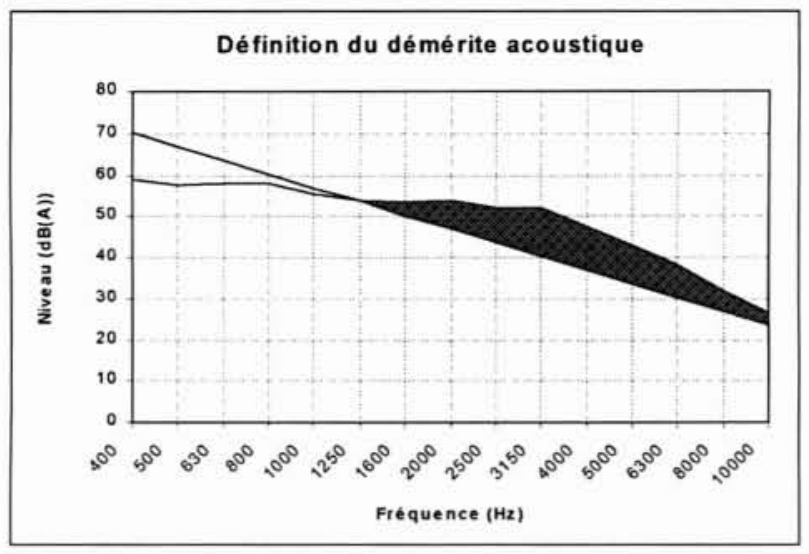

3. Définition du démérite acoustique.

La soufflerie aéroacoustique est un moyen indispensable, puisqu'elle est seule à permettre de faire la synthèse du bruit aérodynamique. On y met en effet au point le bruit de forme et celui des accessoires extérieurs d'une part, de même qu'on valide et finit la mise au point des étanchéités initiée en chambre réverbérante.

La mise au point des bruits aérodynamiques à l'intérieur d'un véhicule fait appel à des mesures qui sont comparées au cahier des charges aéroacoustique, mais aussi nécessairement à des essais d'écoute subjective du spécialiste à l'intérieur du véhicule. Le cahier des charges peut être exprimé sous forme d'un "démérite acoustique" calculé en estimant la surface comprise entre le spectre mesuré et une droite critère (fig. 3).

c) Techniques de mesure et moyens d'essais

Compte tenu des niveaux de bruits aérodynamiques perceptibles à l'intérieur d'un véhicule, il est nécessaire pour les étudier, d'avoir recours à une soufflerie silencieuse telle que le niveau de bruit généré par son fonctionnement soit au moins $10 \mathrm{~dB}$ au-dessous du niveau des bruits aérodynamiques à étudier (Exemple fig. 4).
Le conduit est traité acoustiquement au niveau des angles du circuit, l'hélice est optimisée et les parois de la salle de mesure sont recouvertes d'insonorisant [6]. Elle bénéficie donc d'un bruit de fond particulièrement faible et parfaitement adapté à l'étude des bruits aérodynamiques.

Dans les souffleries les mieux équipées, on peut disposer de moyens de mesure du bruit extérieur généré dans l'écoulement ([1], [3], (4) et [5]) :

- sonde d'intensimétrie,

- miroir acoustique,

- antenne de micros.

L'objectif de telles mesures est d'identifier spatialement les zones générant du bruit et de caractériser les sources en fréquence. Ces techniques peuvent aider la mise au point des accessoires extérieurs en localisant les défauts et en facilitant leur compréhension et leur hiérarchisation.

Ces mesures peuvent être couplées à des explorations aérodynamiques de l'écoulement pour évaluer les vitesses au niveau d'un joint, ou décrire le sillage d'un rétroviseur (taille, orientation, vorticité) par exemple. Ces renseignements complémentaires aux mesures acoustiques peuvent souvent aider au diagnostic et à la découverte de solutions.

\section{III — EXEMPLES DE MISE AU POINT EN BRUITS AÉRODYNAMIQUES}

\subsection{Bruits d'étanchéités}

C'est l'une des contributions les plus importants au bruit aérodynamique perçu dans l'habitacle. Ce type de bruit est à la fois très visible sur les analyses spectrales des enregistrements ( 5 à $10 \mathrm{~dB}(\mathrm{~A})$ Cf. fig. 5), et aussi très perceptible par le passager. La source est en effet monopolaire, elle est créée par les fuites qui peuvent apparaitre lorsque les portes sont déformées par les efforts aérodynamiques.

L'autre contribution que l'on observe au niveau du joint périphérique principal de porte provient non pas de fuites, mais de l'excitation turbulente dans la feuillure. Le fait de lisser le volume avec du ruban adhésif permet d'éliminer cette sollicitation et donc de réduire le bruit intérieur.



4. Plan de la soufflerie aéroacoustique du FKFS de l'Université de Stuttgart 


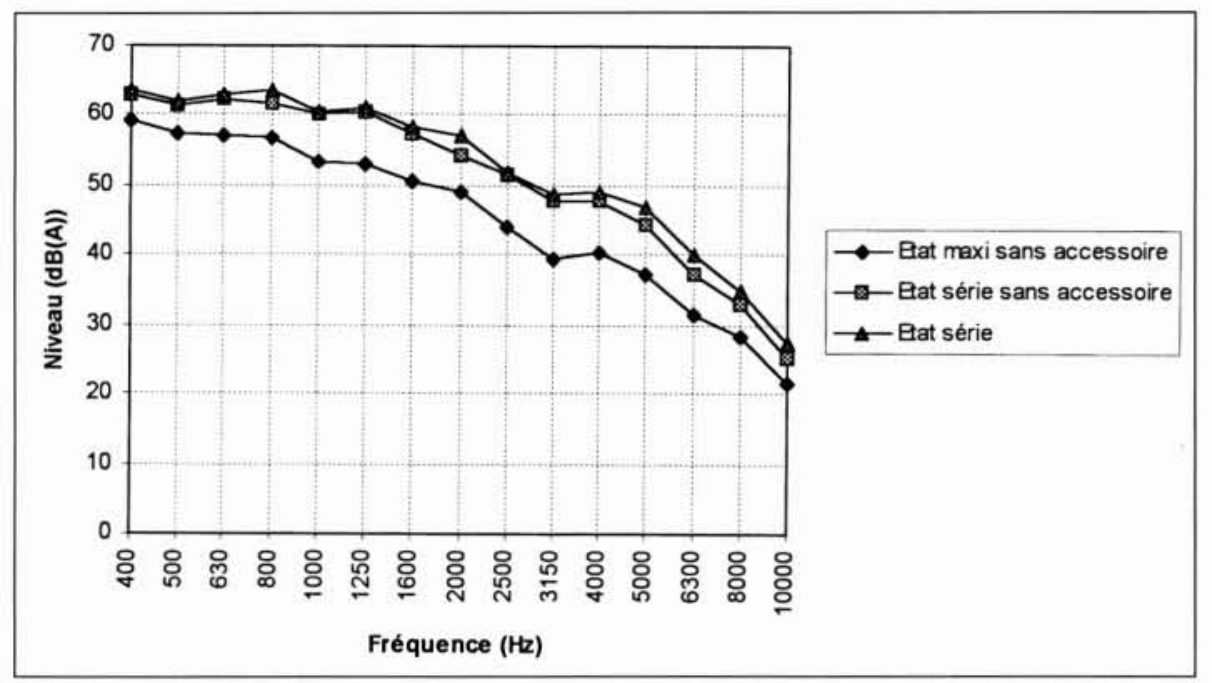

5. Contribution des étanchéités au bruit aérodynamique.

\section{- 3.2 Bruit de forme}

C'est un sujet qui peut être travaillé indépendamment des étanchéités et des accessoires. Le paramètre prépondérant est le rayon du montant de pare-brise (montant A). Il influe non seulement sur le niveau de bruit mais aussi sur la variation de ce dernier en fonction de l'angle de dérapage.

Les figures 6 et 7 indiquent, chiffré en points ( 1 point $=1 \mathrm{~dB}$ par tiers d'octave entre $400 \mathrm{~Hz}$ et $10000 \mathrm{~Hz}$ ), l'effet du rayon sur le niveau de bruit aérodynamique.

Comme on peut le remarquer, c'est sur la sensibilité au dérapage que le rayon est le plus influent. C'est particulièrement ressenti par les passagers lors de dépassement de véhicules en usage autoroutier: la direction du vent apparent varie lors de ces conditions, et peut alors occasionner une forte variation du niveau de bruit intérieur, point jugé négativement en subjectif par des occupants.

\section{- 3.3 Bruit des accessoires extérieurs}

\subsubsection{Mise au point du bruit des essuie-vitres}

Le traitement du bruit des essuie-vitres adopte différentes solutions.

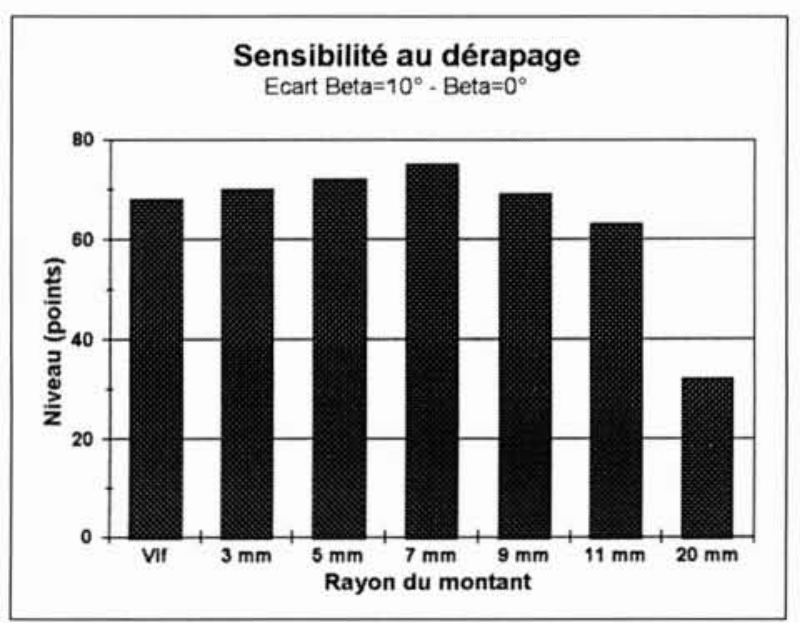

La solution la plus radicale consiste à rentrer le plus possible les essuie-vitres grâce à une position d'arrêt dite "parking " (comme sur Peugeot 605), ce qui représente également un intérêt pour l'esthétique et le confort de vision avant. Cependant, son surcoût par rapport à un mécanisme d'essuie-vitres traditionnel réserve cette solution aux véhicules haut de gamme.

Une autre solution potentiellement sans incidence sur le coût consiste à adapter la géométrie de la fin du capot par rapport à la position basse d'arrêt des essuie-vitres. Cette solution nécessite toutefois de bien anticiper la synthèse entre le style (inclinaison capot pare-brise), le confort de vision vers l'avant, le coût et la faisabilité du capot et des charnières d'articulation. Des évolutions de la base de capot de l'ordre de $10 \mathrm{~mm}$ peuvent permettre une réduction substantielle du niveau de bruit intérieur de l'ordre de 4 à $5 \mathrm{~dB}$ (fig. 8). D'autre part, comme on peut l'observer sur le graphique, une telle modification limite la bosse classique centrée sur $4000 \mathrm{~Hz}$, ce qui améliore fortement la qualité du bruit aérodynamique dans l'habitacle.

\subsubsection{Mise au point des rétroviseurs}

En ce qui concerne les rétroviseurs extérieurs, la réduction des bruits aérodynamiques consiste à optimiser le sillage aérodynamique du rétroviseur en cherchant à atteindre les objectifs suivants :

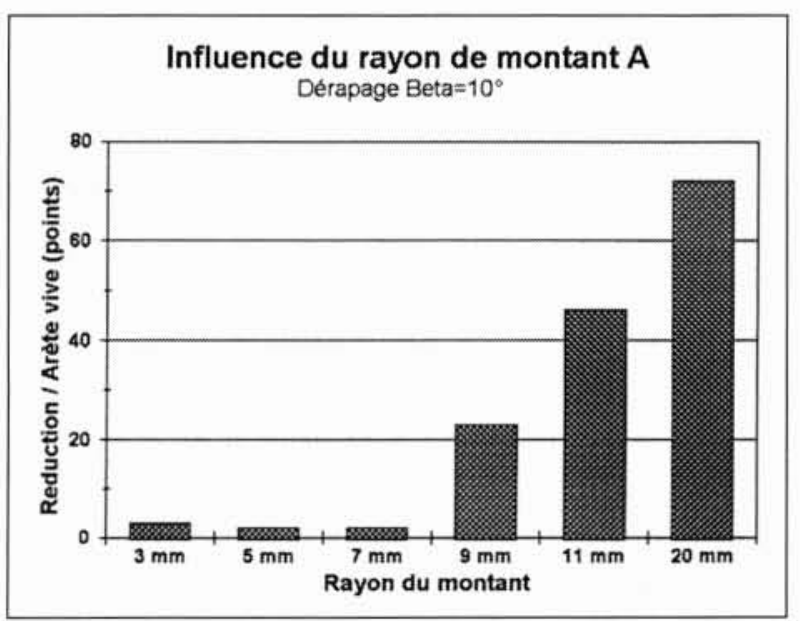

6 et 7. Influence de l'augmentation du rayon de montant A sur le bruit aérodynamique. 


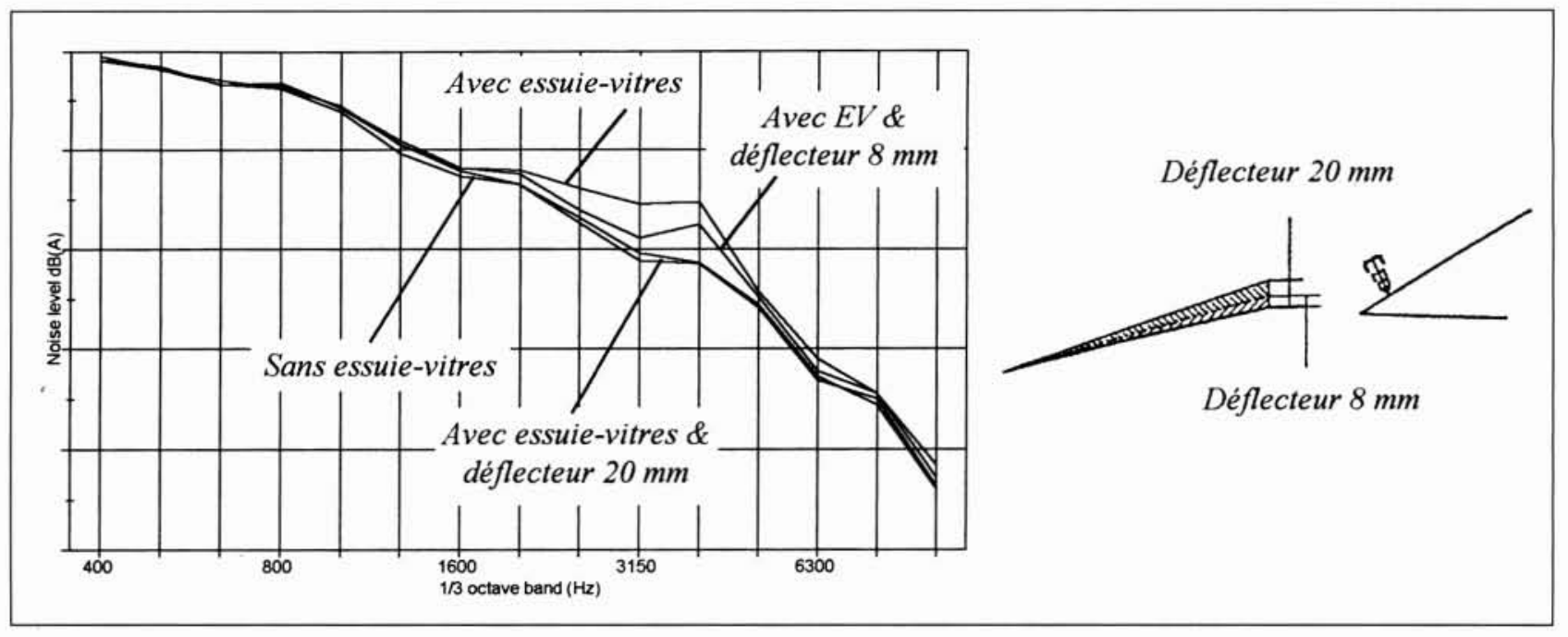

8. Réduction du bruit aérodynamique propre aux essuie-vitres

9. Mise au point d'un rétroviseur

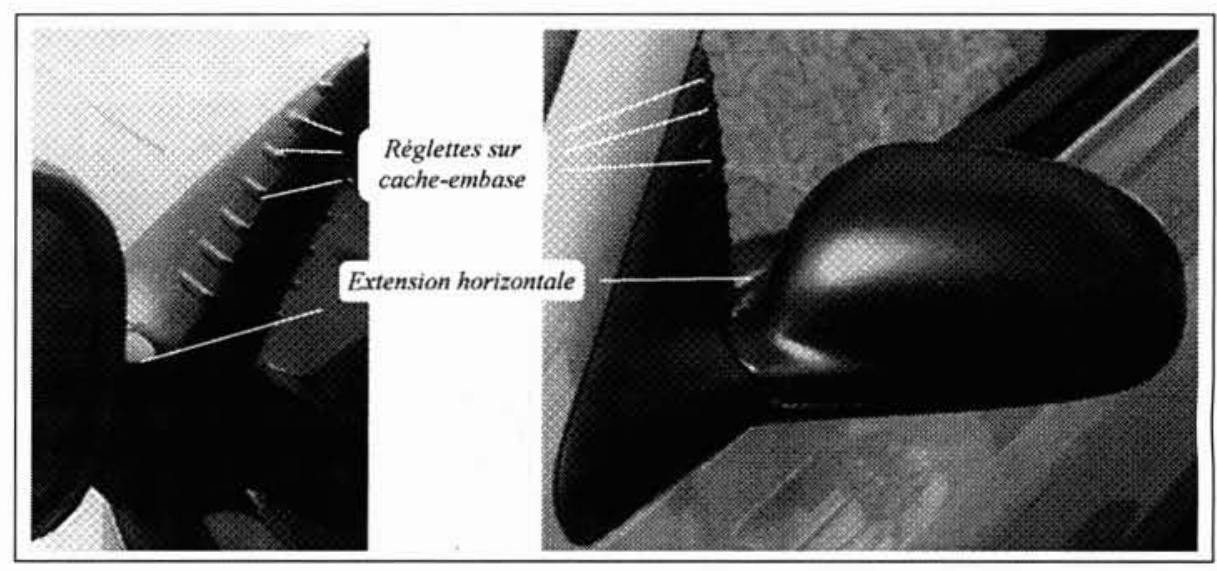

- minimisation de la taille du sillage,

- limitation des fluctuations de l'écoulement pouvant apparaître entre le boîtier et l'embase,

- abaissement de la zone de recollement du sillage du rétroviseur sur le flanc de la voiture,

- élimination des petites recirculations au niveau du boîtier, - élimination des bruits causés par les trous sous boîtier, les fentes et les jeux.

Pour ce faire, il n'existe pas de recette universelle quant à la géométrie des rétroviseurs, les différents types de rétroviseurs (boîtier avec ou sans embase etc.) permettant d'obtenir des performances aéroacoustiques acceptables. Dans le cas, des rétroviseurs de la 406, des nervures ont été créées sur l'embase du rétroviseur et une extension horizontale sur le boîtier près de l'embase (fig.9). Ces deux modifications ont permis une atténuation du bruit propre au rétroviseur atteignant $3 \mathrm{~dB}$ dans certains tiers d'octave.

\subsubsection{Mise au point des antennes de toit}

Le bruit aérodynamique des antennes se caractérise par l'émergence de sifflements caractéristiques qui sont particulièrement visibles sur les spectres en bandes fines. La mise au point porte entre autres sur les paramètres suivants :

- la longueur du brin,

- I'inclinaison du brin,

- la présence d'un ressort en pied de brin.

- la mise en place d'une torsade sur le brin.
Tous ces points ont une grande importance sur l'émergence de pics. La figure 10 met en évidence l'effet de l'inclinaison du brin. Ce graphe correspond à la différence en bandes fines des spectres avec et sans antenne de toit pour une antenne longue standard (sans ressort ni torsade) à l'avant du pavillon.

On peut remarquer que l'effet de l'inclinaison influe à la fois sur le positionnement en fréquence des pics et sur leur intensité. La solution la plus favorable est celle où le brin est le plus incliné.

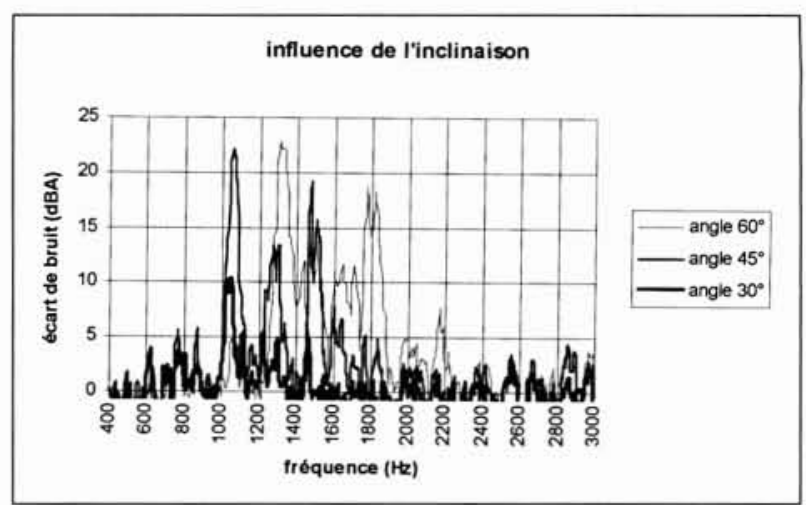

10. Influence de l'inclinaison du brin d'antenne sur les pics de bruit émergents 


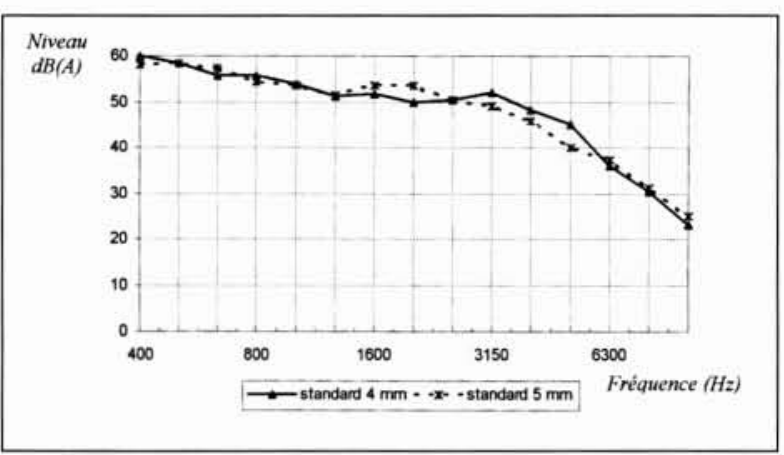

11. Influence de l'épaisseur du vitrage sur le bruit aérodynamique intérieur.

\section{- 3.4 Isolation des vitrages}

Lorsque les contraintes techniques et les choix de style ne permettent pas d'obtenir des rétroviseurs suffisamment silencieux, la seule solution est de diminuer la transparence des vitres latérales. Elle peut l'être de deux manières :

- augmentation de l'épaisseur,

- utilisation de vitrages feuilletés.

Comme le montre la figure 11 , une augmentation d'épaisseur de 4 à $5 \mathrm{~mm}$ permet de réduire de $3 \mathrm{~dB}$ le bruit entre 3000 et $5000 \mathrm{~Hz}$, même si l'on observe une détérioration autour de $1600 \mathrm{~Hz}$. L'inconvénient d'une telle solution est l'augmentation de la masse de la vitre et la persistance d'un spectre possédant une allure médiocre.

La seule solution vraiment efficace est l'emploi de vitrages feuilletés (fig. 12), le gain est alors double :

- réduction du niveau,

- disparition de la faiblesse au niveau de la fréquence de coïncidence.

Il est à noter que ces résultats ont été obtenus avec des vitrages feuilletés standards non acoustiques (type parebrise). Une mise au point du feuilletage permettrait donc d'obtenir des résultats encore meilleurs.

L'activité d'étude des bruits aérodynamiques des véhicules particuliers s'est intensifiée ces dernières années. L'apparition en Europe de souffleries aéroacoustiques performantes adaptées à l'automobile, offrant la possibilité d'étudier les bruits aérodynamiques à tous les stades du développement d'un projet, permettra de réaliser des progrès significatifs dans le domaine du confort acoustique des véhicules particuliers.

D'autre part, les futures avancées les plus significatives porteront sur les méthodes d'analyse par une généralisation de l'utilisation des techniques de psychoacoustique et des studios de réécoute ([7] et [8]).

Enfin, bien qu'essentiellement expérimentale, la mise au point des futurs véhicules intégrera de plus en plus de résultats numériques, seuls moyens permettant de réduire encore les délais de conception de nos véhicules.

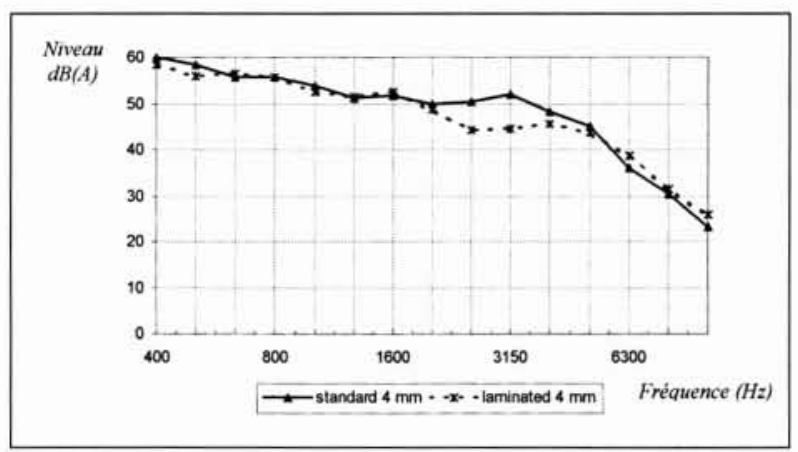

12. Influence du type de verre sur le bruit aérodynamique intérieur.

\section{RÉFÉRENCES}

[1] GEORGE A.R., CALLISTER J.R., 1996, Aeroacoustics of passenger cars - State-of-the-art, Seminar Aerodynamics and powertrain automobile noise - Aeroacoustics - 1996. Pininfarina - PF/CNR SP 9601

[2] ZACCARIOTTO M., BURGADE L., CHANUDET P., 1997, Aeroacoustic studies at P.S.A., 2. Stuttgarter Symposium - Kraftfahrwesen und Verbrennungsmotoren, expert verlag, ISBN 3-8169-1522-1, 511-518

[3] BURGADE L., 1998, Introduction à l'aéroacoustique automobile, Cours C.L.E.S.I.A., Société des ingénieurs de l'automobile, Session 97-98

[4] COGOTTI A., 1996, Recent developments on aeroacoustics at Pininfarina, Seminar Aerodynamics and powertrain automobile noise - Aeroacoustics -1996, Pininfarina - PF/CNR SP 9601

[5] HELFER M., MELCHGER N., BUSCH J., 1996, Moyens de mesure pour les bruits aérodynamiques intérieurs et extérieurs de véhicules, Congrès S.I.A. Aérodynamique - Aéroacoustique-Aérothermique Automobile et Ferroviaire, S.I.A. 96.09 .06

[6] POTHOFF J., HELFER M., ESSERS U., 1994, The new aeroacoustic windtunnel at Stuttgart university - Equipment and first results, Actes de la conférence S.I.A. Dynamique du véhicule - Lyon 8-9 Juin 1994

[7] CAMPINO S., 1996, Mesures acoustiques : dépassez la notion de bruit, misez sur la qualité acoustique, Mesures 687 - Septembre 1996, 53-56

[8] ZWICKER E., FASTL H., 1990, Psycoacoustics - Facts and models, Springer 